\title{
Die Besonderheiten der Wasserströmung in phy- sikalischer, chemischer und biologischer Hinsicht
}

\author{
Von H. АмвӥHL
}

(Eidgenössische Anstalt für Wasserversorgung, Abwasserreinigung und Gewässerschutz an der Eidg. Technischen Hochschule, Zürich).

Was die ökologische Wissenschaft so kompliziert und schwierig macht und manchen Biologen davon abhält, sich ökologischen, zumindest synökologischen Fragen zu widmen, ist die Vielfalt der wirksamen Faktoren, jenes so überaus schwer zu überblickende Netzwerk von eigenständigen, von sich gegenseitig beeinflussenden und auch von eng zusammenhängenden Ursachen, Wirkungen und Wirkungsmechanismen. Freilich sei damit nicht gesagt, dass die Fliesswasserbiologie hier besonders im Nachteil ist - gewiss hat die Seenkunde, um das uns wohl am nächsten liegende Beispiel heranzuziehen, auch ihre Sorgen und Nöte diesbezüglich. Indessen läuft man hier wie dort gar leicht Gefahr, einen einzigen Faktor, weil man ihn durch fortgesetzte Analysen einigermassen kennengelernt hat, zu überschätzen, und vernachlässigt darob die andern Teile des Ökosystems, um dessen gesamthaftes Erkennen es ja letztlich geht.

Eine Reihe von Fachleuten hat sich hier zu einem Symposium zusammengefunden, um einen dieser ökologisch wirksamen Faktoren $\mathrm{zu}$ besprechen, nämlich die Bewegung des Wassers. Zwar wird niemand in Abrede stellen wollen, dass die Strömung nicht ein Faktor ist, der in der Ökologie der fliessenden Gewässer, vom Bergbach bis zum langsam verebbenden Strom des Tieflandes, eine ganz hervorragende Stellung einnimmt und an Wichtigkeit manche anderen, sonst sicher nicht unbedeutenden Faktoren, darunter die Temperatur und den natürlichen Sauerstoffgehalt des Wassers, zu übertreffen scheint [I], doch wollen wir darob in Konsequenz des Gesagten nicht vergessen, dass auch die Strömung im Gefüge der ökologischen Faktoren nur ein einziger Teil ist, der aber, um sinnvoll in den biologischen Rahmen eingefügt zu werden, eben auch der andern Teile bedarf. In diesem Zusammenhang mag man sich der Warnung MacaNs [5] erinnern, extremen Verhältnissen nicht eine zu grosse Bedeutung zuzumessen. 
Sicher ist es nicht überflüssig, wenn wir uns zu Beginn des Symposiums darüber Rechenschaft geben, wie die Strömung als physikalische Erscheinung beschaffen ist (Turbulenz), welche Besonderheiten sie auszeichnen (Grenzschichten und Totwässer) und schliesslich, welche Schlussfolgerungen biologischer Art daraus zu ziehen sind. Zwar geschieht dies hier nicht zum ersten Mal; diese Gedanken sind schon an verschiedener Stelle publiziert worden $[\mathbf{I} ; 6]$. Weil sie aber doch einigermassen neu sind, mag es einen Sinn haben, dass das Symposium mit einer kurzen Auseinandersetzung über die physikalische Seite des bewegten Wassers begonnen wird.

\section{Die wichtigsten Kennzeichen des bewegten Wassers}

"Was die Einzigartigkeit der Fliessgewässer als Lebensraum bedingt, ist, dass das Medium hier in ständiger einsinniger Bewegung und damit in ständigem Wechsel und ständiger Erneuerung begriffen ist. Gefälle, Wasserführung und Untergrund sind in gleicher Weise an dem Zustandekommen dessen, was man die «Strömung» zu nennen pflegt, beteiligt. Die Strömung greift in alle Bereiche des Lebens im Fliessgewässer ein!» (G. PLeskot [6]). Bevor wir uns jedoch in diese Bereiche des Lebens vorwagen, sei die Strömung als solche genauer analysiert. In manchen Teilen mag das als eine Wiederholung bekannter Tatsachen empfunden werden; diese sind aber für das spätere Verständnis der biologischen Erscheinungen im Fliesswasser allzu wichtig, als dass sie an dieser Stelle übergangen werden dürften.

\section{Die laminare und die turbulente Strömung}

An den Anfängen der Strömungslehre steht das bekannte Gesetz von HAGEN-Poiseuille. Dieses sagt unter anderem aus, dass sich die Flüssigkeit, welche durch ein rundes Rohr strömt, nicht gleichförmig bewegt, sondern dass die zur Achse parallelen Geschwindigkeitsvektoren so verteilt sind, dass die höchste Geschwindigkeit in der Mitte herrscht, an jenem Punkt somit, der von der Wandung am weitesten entfernt ist, und die Geschwindigkeit gegen die Wandung zu im Quadrat dieser Entfernung abnimmt. Unmittelbar an der Rohrwand herrscht Ruhe. Auf allen zur Rohrachse konzentrischen Wasserschichten herrscht jeweils die gleiche Geschwindigkeit; diese einzelnen Flüssigkeitszylinder gleiten gewissermassen nebeneinander her, frei von Durchmischungsvorgängen. 
Diese Bewegungsweise ist bekannt als die laminare Strömung. Man findet sie am ehesten dort, wo ein Wasserstrahl aus einem Rohr austritt, bei einem Wasserhahn zum Beispiel. Solange der herabfallende Strahl klar, glasig und ungestört ist, handelt es sich um laminare Strömung. Sie ist umso stabiler, je dünner der Strahl selber ist und je langsamer das Wasser fliesst.

In der Natur findet sich laminare Strömung praktisch nur im Grundwasser; unter den Bedingungen des kapillaren Fliessens, bei den hier herrschenden sehr geringen Geschwindigkeiten bleibt laminare Strömung stabil, während sie in Oberflächengewässern kaum existieren kann:

Sobald nämlich das laminar fliessende Wasser eine gewisse Geschwindigkeit erreicht, zerfällt die geordnete Strömung; die Wasserfäden und Wasserschichten, die vorher, ohne sich gegenseitig zu stören, nebeneinander her geflossen sind, lösen sich auf; Unebenheiten in der Wandung und an der Sohle des Gerinnes haben Störungen im wandnahen Strömungsverlauf zur Folge, was zur Ausbildung von Wirbelablösungen und damit von Wirbeln führt. Diese bewirken ihrerseits in ihrer Gesamtheit, in eine unübersehbare Fülle einzelner Bewegungen zerlegt, die Turbulenz. Turbulenz bedeutet nun aber nicht einfach wirblig bewegtes Wasser - es gibt schliesslich auch in der laminaren Strömung wirbelähnliche Erscheinungen -, sondern turbulentes Wasser teilt sich gewissermassen in einzelne dauernd wirblig durcheinandermischende Wasserpakete auf, deren Grösse und Geschwindigkeit durch den Grad der Turbulenz bestimmt ist.

Der Punkt, bei dem die laminare Geschwindigkeit in die turbulente übergeht, ist für ideale Gerinne angegeben worden, und zwar hängt er von einer dimensionslosen Grösse $a b$, der Reynoldschen Zahl, welche sich aus der Geometrie der verwendeten Apparatur und den Eigenschaften der Flüssigkeit berechnen lässt. (Einzelheiten darüber zum Beispiel bei [7]). Erreicht diese Zahl nun eine bestimmte Grösse, so zerfällt die laminare und wird zur turbulenten Strömung. Die tatsächlichen Verhältnisse sind hier nicht einfach. So gibt es keine universelle kritische Reynoldsche Zahl, sondern ihr Betrag hängt in einem weiten Bereich von den äusseren Bedingungen $\mathrm{ab}$. Immerhin sieht man aus der Reynoldschen Formel, dass die Geschwindigkeit, bei der dieser Wechsel stattfindet, umso kleiner ist, je grösser das Gerinne ist; sie ist dem Durchmesser der Röhre zum Beispiel umgekehrt proportional. In offenen Gerinnen wird sie nochmals um die Hälfte kleiner. Bei einem Rohr von I cm Durchmesser liegt die kritische Geschwindigkeit unter idealen Bedingungen bei $18 \mathrm{~cm} / \mathrm{s}$, in einem Rohr mit I $m$ Durchmesser fällt sie dagegen auf $\mathrm{I}, 8 \mathrm{~mm} / \mathrm{s}$, und 
in einem offenen Gerinne mit I $m$ Breite fällt sie weiter $a b$ bis unter I $\mathrm{mm} / \mathrm{s}$. Eine solche Geschwindigkeit wäre in einem Oberflächengewässer schon kaum mehr wahrnehmbar. So versteht man auch, dass es in den Oberflächengewässern eine rein laminare Strömung nicht gibt, auch nicht in grossen Flüssen, ja nicht einmal in Seen, weil bei solch grossen Wassermassen die Reynoldsche Zahl auch bei geringsten Geschwindigkeiten schon weit über dem kritischen Wert und damit über der kritischen Geschwindigkeit liegt. Auch die Zirkulationsströmungen in den Seen gehen turbulent vor sich: sie wären anders überhaupt gar nicht denkbar. Wenn wir deshalb im folgenden von «Strömung» sprechen, so ist durchwegs turbulente Strömung gemeint. Eine Ausnahme freilich wäre zu machen bei einer Betrachtung der hydraulischen Verhältnisse des Psammons. Hier, in diesem ganz besonderen Lebensraum, werden zur Hauptsache die Verhältnisse der laminaren Strömung herrschen, welche aber, da sie sich in einem komplizierten Gitterwerk von Hohlräumen und Lücken zwischen den Sandkörnern bewegen muss, dauernd aufgeteilt und während des Weiterfliessens neu zusammengefügt wird. Sie nähert sich in einem gewissen Grade einer vereinfachten Turbulenz und erfüllt so wenigstens annähernd die Funktion der turbulenten Strömung, nämlich den Wasserund Stoffaustausch, wenn sie auch physikalisch nicht die Bedingungen der Turbulenz einhält.

Wozu diese lange Erklärung? Die Turbulenz ist die mechanische Grundlage für den Stoffaustausch im Wasser, nicht nur im See, sondern auch im fliessenden Wasser. Durch die besonderen Verhältnisse der 'Turbulenz wird die bewegte Wassermasse dauernd homogenisiert; lokale Ungleichheiten ihrer Salzkonzentration und ihres Sauerstoffgehaltes werden ausgeglichen, und dies fast nur auf rein mechanischem Wege. Die Diffusion spielt gerade im fliessenden Wasser eine recht bescheidene Rolle. Der turbulente Austausch greift aber auch in den Seen, ja sogar im Meer bis in die grössten Tiefen hinunter, was denn auch GessNer zum Ausspruch veranlasst, dass das Wasser ohne Turbulenz «kein Lebensraum mehr» wäre, «sondern ein Bereich des Todes» [3, Seite 20I]. Ohne Wasser kein Leben - ohne Turbulenz kein Leben im Wasser!

Die Turbulenz ist somit die physikalische Erscheinung, die Ursacbe, der Austausch ist die Wirkung. Dass Gewässer mit starker Turbulenz, das heisst mit einem sehr regen Stoffaustausch dichter besiedelt werden können, ist eine der Folgerungen daraus. Zwar ist die im allgemeinen überraschend dichte Besiedlung steiniger Bachsohlen nicht allein auf diese Erscheinung zurückzuführen; manche noch unbekannten Faktoren 
des Verhaltens von Tieren und Pflanzen werden hier mit hineinspielen, doch hat daran die turbulente Strömung mindestens einen wesentlichen Anteil. Auch der von RUTTNER so treffend formulierte Begriff des «physiologischen Sauerstoffreichtums» des fliessenden Wassers ist eine direkte Folgeerscheinung der Turbulenz bzw. des Austausches [8;9 (Seite 263)].

Zwischen zwei zusammenfliessenden Wassermassen vollzieht sich der Austausch wohl ziemlich unbehindert (im See beispielsweise), da dort keine festen Hindernisse vorhanden sind. Gilt es aber, das Wasser, welches einen festen Körper, ein Tier oder eine Pflanze umgibt, zu erneuern, so stossen wir auf eine andere, ebenso wichtige physikalische Erscheinung, welche den Austausch in gewissen Grenzen erschwert, nämlich die

\section{Grenzschichten [boundary layers, couches limite]}

Grenzschichten nennen wir jene Schicht von Flüssigkeit, die infolge der inneren Reibung oder der Zähigkeit des Wassers in der Nähe jedes ruhenden Körpers abgebremst wird. In dieser dünnen Schicht steigt die Geschwindigkeit von Null am Substrat rasch auf den Wert der mittleren Aussenströmung an. In der Technik spielt diese Grenzschicht eine grosse Rolle; deshalb ist es eigentlich zu verwundern, dass man sie in der Biologie so lange wohl am Rande erwähnt, daneben aber doch ziemlich vernachlässigt hat. Sie ist aber biologisch durchaus bedeutsam und nimmt innerhalb des Komplexes der Strömungsphänomene eine ausserordentlich bedeutsame Stellung ein.

Auch hier schliessen wir uns voll und ganz der Meinung von G. PLESKOT an, wonach zur Untersuchung des «Kleinklimas» der Wasserströmung in Bodennähe bisher die geeigneten Messeinrichtungen gefehlt haben [6]. Sie fehlen auch heute noch. Es ist auch heute noch nicht möglich, die Wassergeschwindigkeit in der unmittelbaren Nähe des Substrates in einem Bach genau zu messen, wenigstens wenn man von einem neuen englischen Gerät absieht, dessen Flügelrad mit einem Durchmesser von einem einzigen Zentimeter erlaubt, schon recht nahe heranzukommen [2]. Ein $\mathrm{cm}$ ist aber in den meisten Fällen noch zu weit entfernt von der besiedelten Wasserschicht, so dass wir uns weiterhin darauf beschränken müssen, aus geeigneten Modellversuchen auf die tatsächlichen Verhältnisse in Fluss und Bach zu schliessen. Das wird auch für die nächste Zeit noch unser Arbeitsverfahren bleiben müssen, solange zumindest, bis sich jemand aus der Technik der Strömungsmessung für die besonderen Zwecke der Biologen annimmt. Das Fehlen geeigneter Me- 
thoden ist ja nicht nur hier, sondern leider auch in andern Sparten der Limnologie ein eigentlicher limiting factor.

Was die Grenzschichten anbetrifft, so haben wir uns vor einigen Jahren bemüht, in Ermangelung solcher Messeinrichtungen die Strömungsverhältnisse owenigstens im Modell, in künstlichen Kanälen im Laborversuch zu reproduzieren. Wir verwendeten dafür das Verfahren des optischen Schnittes: Aufgeschwemmte Partikel eines weissen Materials (Azetylzellulose) werden durch einen intensiven Lichtspalt sichtbar gemacht und geben die Strömung in der Rinne derart klar wieder, dass es ohne weiteres möglich ist, auf Photographien auch die Grenzschicht auszumessen [I]. Dabei mag uns trösten, dass auch in der zünftigen Hydraulik die Messung der Grenzschichten keine einfache Sache ist.

Die Grenzschicht selber ist nun im Gegensatz zu manchen in der Literatur geäusserten Ansichten kein starres oder völlig unbewegtes Gebilde; sie ist lediglich jene Zone, jener Hof rund um den betreffenden Körper herum, in dem die Geschwindigkeit des vorbeiziehenden Wassers stark verlangsamt ist. Für einige Spezialfälle hat man den Verlauf der Grenzschichten mathematisch gefasst; diese Ableitungen sind aber sehr kompliziert. Einzig der einfachste Fall der Grenzschicht längs einer geraden Platte mag uns dartun, in welcher Weise sich die Grenzschicht etwa entwickelt. Definiert als die Zone verlangsamter Strömung, vollzieht sich ihr Übergang zum freien Wasser nicht plötzlich, sondern stetig. Sie ist selber ein fliessendes Gebilde, in dessen Innerem freilich meistens laminare Verhältnisse herrschen. Ihre Dicke hängt unter anderem von der Länge der angeströmten Platte und von der Fliessgeschwindigkeit des Wassers ab (Abb. I), und zwar ist sie proportional zur Wurzel aus dem reziproken Wert von Länge und Geschwindigkeit.

Dieses in Abbildung I schematisch dargestellte, weitgehend theoretische Strömungsbild genügt aber für das Verständnis der Strömungsverhältnisse
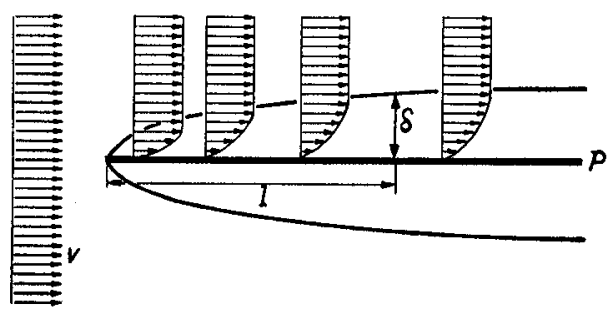

Abbildung 1

Schematische Darstellung der Grenzschicht an einer längs angeströmten Platte. $v$ Geschwindigkeit; $l$ Abstand des Messpunktes von der Plattenvorderkante; $\delta$ Dicke der Grenzschicht. 


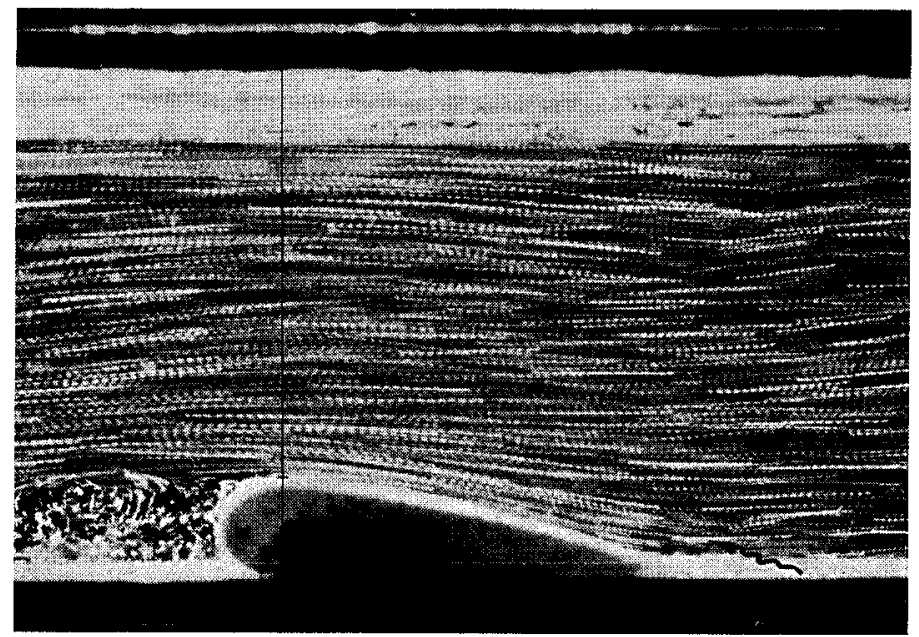

Abbildung 2

Verlauf der turbulenten Wasserströmung um einen Modellstein herum. In der Nähe des Substrates ist die Geschwindigkeit deutlich vermindert (gewellter Pfeil).

an der natürlichen Gewässersohle nicht. Eine konkretere Anschauung vermitteln Bilder, welche mit der erwähnten optischen Einrichtung gemacht wurden und die Strömung direkt wiedergeben (Abbildung 2). Es handelt sich hier um Momentaufnahmen; unregelmässige Geschwindigkeit der vorbeifliessenden Tracer-Partikel sind das Kennzeichen starker Turbulenz. Um aus solchen Bildern den Strömungsverlaut genau auszumessen, mussten alle diese Aufnahmen mehrmals wiederholt werden, und erst das Mittel aus allen graphischen Auswertungen in der Art von Abbildung 3 ergab sodann das gewünschte Resultat. Abbildung 4 zeigt den Verlauf der Grenzschicht an einem Modellstein, wie er in jedem Bach natürlicherweise vorkommen kann. Der Stein ist überzogen von einer Zone verlangsamter Strömung, deren Verlauf den gewohnten Regeln der Hydraulik folgt.

Reihenmessungen an solchen Versuchskörpern haben ergeben, dass die Grenzschicht, die sich an einem frei überströmten Körper ausbildet, eine Dicke in der Grössenordnung von Millimetern, etwa I bis $3 \mathrm{~mm}$ besitzt, auch unter extremen Verhältnissen. Dadurch liegt die Grenzschicht tatsächlich in einem Bereich, der von der Körperhöhe der meisten Fliesswassertiere nicht überschritten wird.

Damit kommen wir zur biologiscben Bedeutung der Grenzscbicbt. Vorerst muss aber nochmals betont werden: Die Grenzschicht ist kein stagnie- 


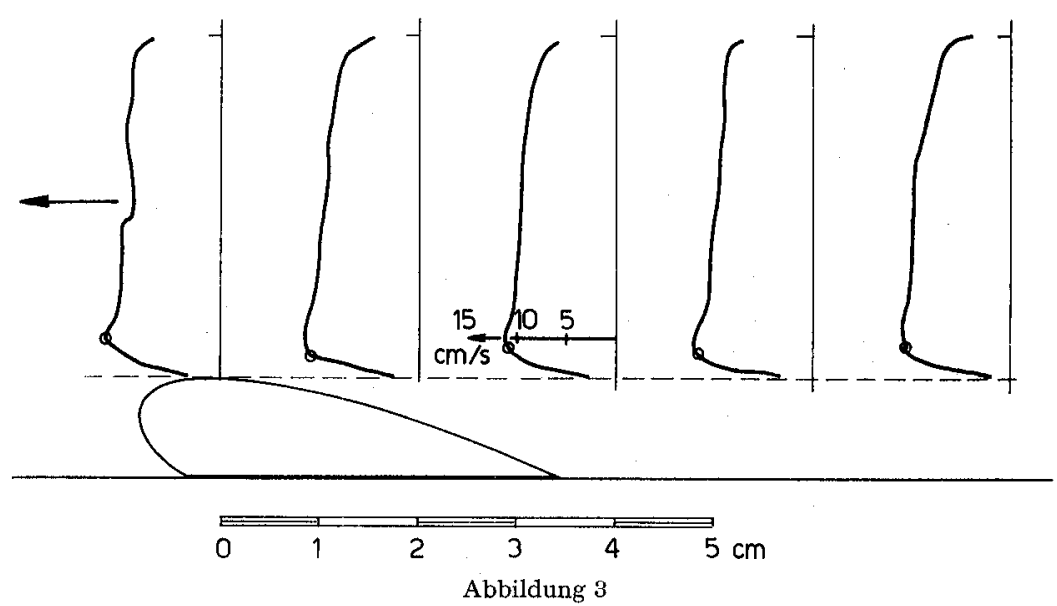

Graphische Auswertung einer Serie von fünf unter gleichen Bedingungen gemachten Momentaufnahmen (Expositionszeit $1 / 5$ Sekunde). Der Übergang von der Grenzschicht zur Aussenströmung ist mit Kreisen bezeichnet. Abbildung 2 ist eine der hier ausgewerteten Aufnahmen.
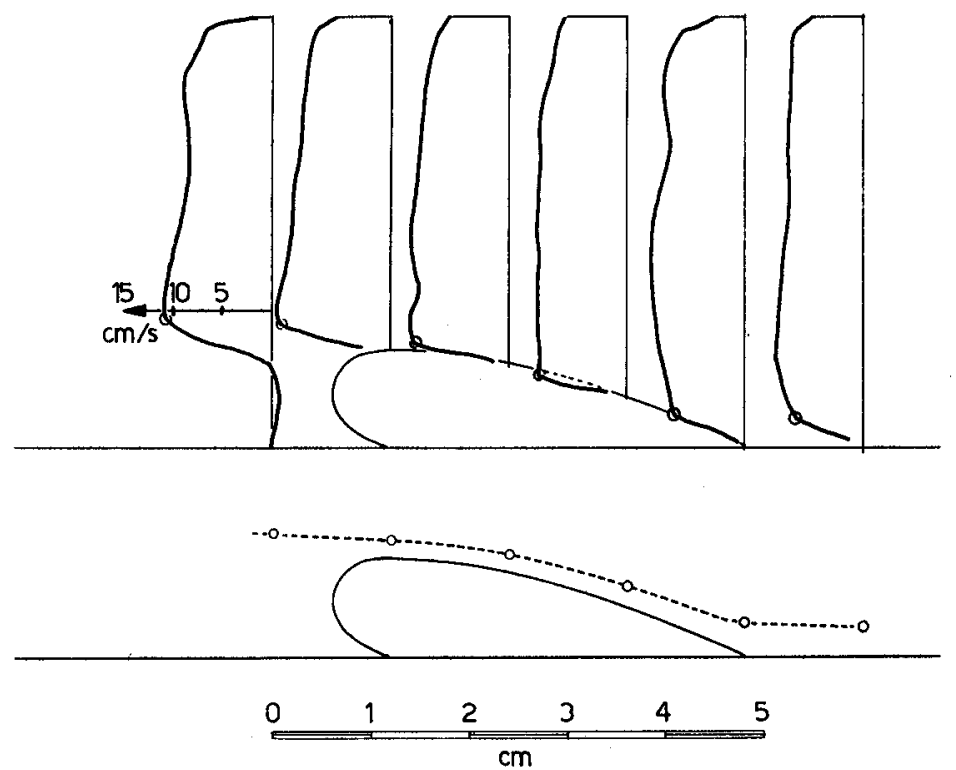

Abbildung 4

Momentane Verteilung der turbulenten Strömung am Modellstein aus Abbildung 2 (graphische Auswertung; oberes Diagramm) und Verlauf der Grenzschicht (unteres Diagramm).

rendes Gebilde, kein Elysium, das jeden Stein im Bach überzieht und in dem sich von jedem äusseren Einfluss ungestört dahinleben lässt, sondern lediglich eine Zone, in der die Unbilden der Strömung gemildert sind. Um so 
mehr gemildert zwar, je näher sich ein Organismus dem Substrat anschmiegen kann, das heisst, je kleiner er ist; einen absoluten Schutz vor der Strömung gibt es aber in der Grenzschicht nicht. Immerhin scheint sie einer Anzahl von lithophilen Bachtieren, namentlich Ephemeropterenund Trichopterenlarven und -nymphen guten Schutz zu bieten. Das erklärt denn auch, wieso ein Teil der Bachtiere, und zwar namentlich jene, welche sonst über keine besonderen Anpassungsmerkmale verfügen, durch besonders kleinen Körperbau auffallen. Ihre Kleinheit ist ihr Merkmal: Die Grenzschicht schützt sie vor der Strömung. Daneben sind aber auch die grösseren Bachtiere durchaus Nutzniesser des «gemässigten Mikroklimas» in Substratnähe.

Das schönste Beispiel liefern hierzu die abgeflachten Larven von Epeorus, Rhithrogena, Ecdyonurus usw. Ob sie sich mit ihrer Körperunterseite am Substrat ansaugen können oder nicht, ist in diesem Zusammenhang ziemlich belanglos; wesentlich ist; dass sie sich so tief an das Substrat anschmiegen, dass die Strömung die Unterseite ihres Körpers nicht mehr erreichen kann und uiber sie hinweggeht. Dieses Verhalten erklärt denn auch die überraschende Beweglichkeit dieser Tiere selbst in stark strömendem Wasser. Durch den flachen Bau ihres Körpers werden sie, hydraulisch gesehen, zu einem Teil des Substrates; sie liegen gewissermassen in der Grenzschicht drin, während diese über sie hinwegzieht. $\mathrm{Zu}$ erwähnen sind in diesem Zusammenhang auch die Massen von Eilarven zum Beispiel von Bxetis verschiedener Arten, welche sich auf den nackten Steinen des Baches aufhalten und hier offenbar in extremer Weise durch die Grenzschicht davor geschützt sind, weggespïlt zu werden.

Es ist hier nicht der Ort, sich mit biologischen Einzelheiten auseinanderzusetzen. Immerhin sei im Zusammenhang mit der Grenzschicht auf die Besiedlung nackter Felsen an Wasserfällen zum Beispiel, auf Steinen in Flüssen oder an überströmten Felswänden im Gebirge hingewiesen. Eine Besiedlung solch extremer Standorte durch Blaualgen, Grünalgen und Moose, zum Beispiel durch Rhodoplax Schinzii an besonders exponierten Stellen im Rheinfall bei Schaffhausen [4] ist überhaupt nur dann denkbar, wenn man das Vorhandensein einer Grenzschicht, das heisst einer verlangsamten Wasserschicht und eines ruhenden Filmes voraussetzen kann.

Damit finden wir, dass die Grenzschicht, als eine durchaus normale und überall vorhandene Naturerscheinung, für die gesamte nicht planktische Lebewelt des Wassers eine fundamentale Bedeutung als mechanischer Schutz vor der Wasserbewegung besitzt, daneben aber auch ein Hindernis darstellt für den Stoffaustausch. 


\section{Das Totwasser [dead water, eau morte, eau calme]}

Es sind grundsätzlich drei Erscheinungen, welche das Bild des strömenden Wassers in einem natürlichen Gerinne prägen:

I. die Turbulenz, als allgemeine Erscheinung,

2. die Grenzschichten und

3. das Totwasser.

Diese letzte Erscheinung ist eigentlich so selbstverständlich, dass man sie kaum mehr besonders erwähnen müsste; sie hat aber für das Leben im bewegten Wasser, namentlich im Bach und Fluss eine allzu hohe Bedeutung, als dass wir sie hier übergehen dürften.

Hinter jeder Erhebung, die ins fliessende Wasser hineinragt, oder hinter jeder Verengung des durchflossenen Profils bildet sich eine Zone aus, in der sich das Wasser nur langsam, im Idealfall als sogenannte Walze (Abbildung 5) bewegt. Wie sie entsteht, können wir uns mit dem folgenden allgemeinen Fall vergegenwärtigen: Verengt sich ein durchflossenes Gerinne, so verändert sich natürlich die Verteilung der Geschwindigkeit; die Randzone und damit die Grenzschicht wird dünner (Abbildung 3 in [I]), das Geschwindigkeitsprofil ist stabil. Erweitert sich der durchflossene Querschnitt etwas, so spitzt sich das Geschwindigkeitsprofil zu; die Randzone wird auseinandergezogen, und nur in der Mitte bleibt die ursprüngliche Geschwindigkeit erhalten. Dieser schon ziemlich labile Zustand bleibt bis zu einem Öffnungswinkel von einigen Graden erhalten; erweitert sich der Kanal noch stärker, so zerfällt die Strömung an dem Punkt, von dem aus die Kanalwände zu divergieren beginnen. Es bilden sich Wirbelablösungen aus, die vorerst in der sogenannten Trennfläche, einer scharf begrenzten Zone, zusammenbleiben, und diese Trennfläche bildet ihrerseits die Grenze zwischen der bewegten, mittleren und den seitlichen, ruhenden Wassermassen, den Totwässern.

Der analoge Fall tritt ein, wenn das durchflossene Gerinne zum Beispiel durch Steine gestört wird, welche auf der Sohle des Baches liegen. Bis zum Scheitelpunkt des Steins herrscht ungestörte Strömung, doch hier tritt Ablösung ein, und die Zone hinter dem Stein ist von der freien Strömung durch die Trennfläche abgetrennt. Sie befindet sich freilich nicht etwa in Ruhe; die Wirbel der Trennfläche liefern genügend Energie, um das Wasser dauernd in mässiger Bewegung zu halten. Vergegenwärtigt man sich, wie die Sohle der Fliessgewässer beschaffen ist, nämlich in der Regel als Sammelsurium der verschiedensten Unebenheiten, von Steinen, Sand- und Kieshäufchen, Schlammbänken, Pflanzenbüscheln 


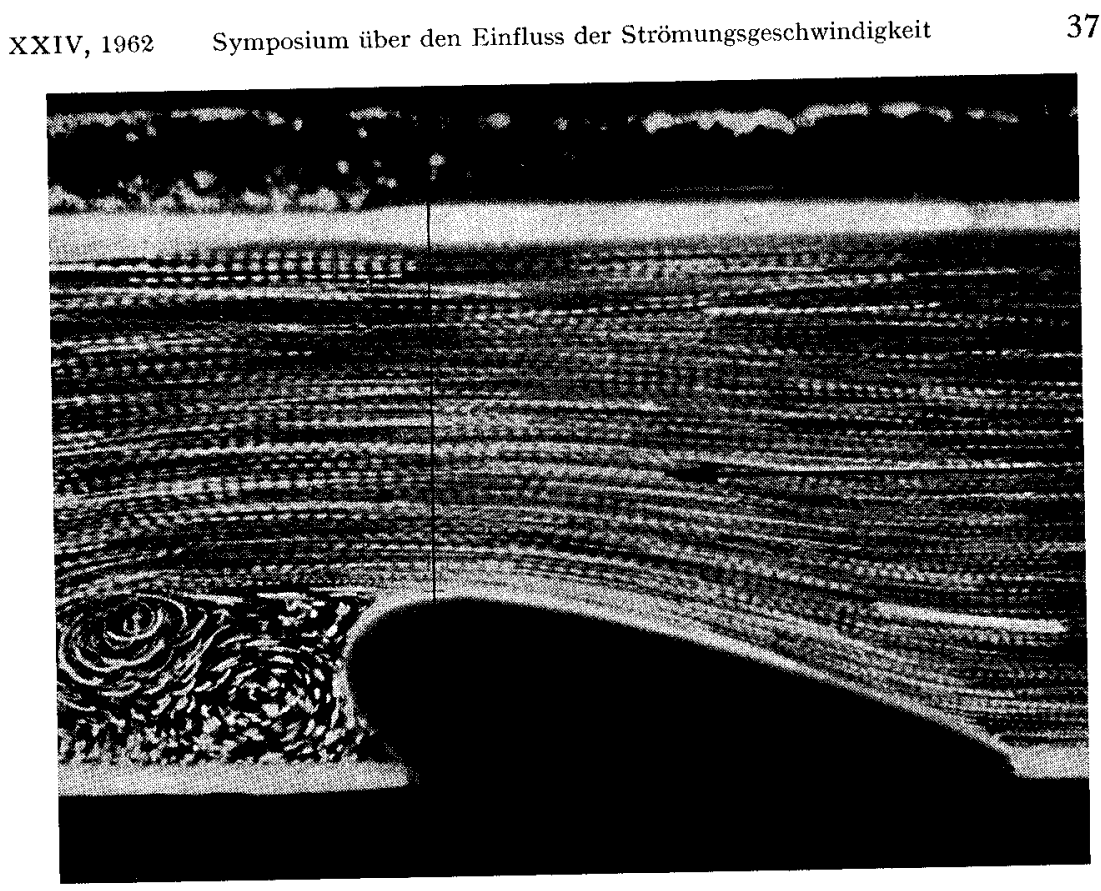

Abbildung 5

Ausbildung der Totwasserzone hinter einem umströmten Modellstein.

usw., von denen jedes seine eigene Totwasserzone ausbildet, so erkennt man, dass die bodennahe Schicht, auch wenn wir von den Grenzschichten absehen, uiberhaupt zum grössten Teil nicht mehr zum eigentlich strömenden Wasser gerechnet werden darf, sondern ein Lebensraum für sich ist. Und damit wird auch verständlich, dass man vielfach auch in starker Strömung Arten finden kann, die eigentlich nicht in einen solchen Lebensraum passen, Gammarus zum Beispiel. Dieser Umstand ist bei ökologischen Arbeiten im fliessenden Wasser unbedingt zu beriicksichtigen, zumal es namentlich unter den Insektenlarven ausgesprochene Totwasserbewohner gibt und daneben Arten, welche sich eindeutig der direkten Wasserströmung exponieren. Auf keinen andern Teil des Lebensraums des fliessenden Wassers trifft der Begriff des «Kleinklimas der Wasserströmung» [6] so gut zu wie auf das Totwasser. Dieser Lebensraum, der einen grossen Teil des gesamten Habitats einnimmt, ist vor dem darüber hinweg fliessenden Wasser derart gut geschützt, ohne aber auf die physiologischen Vorteile des fliessenden Wassers verzichten zu müssen, dass man ihn wohl als wichtigster. Teil des bewohnbaren Fliesswassers betrachten muss. 
Totwässer finden wir aber nicht nur hinter den Steinen der Bachsohle oder in den Zwischenräumen des Kiesbettes, um nur diese zwei Beispiele zu nennen, sondern auch ganz besonders schön ausgebildet im Innern von Pflanzenbeständen, Moos- und Algenbüscheln. Die sogenannte Moosfauna ist hydraulisch gesehen eine Totwasserfauna. Leider fehlen hier aber experimentelle Arbeiten.

Gesamthaft betrachtet, ist somit das Fliesswasser in physikalischer Hinsicht kein unwirtlicher, schwierig zu bewohnender Lebensraum, der nur von besonderen Spezialisten besiedelt werden kann, wie das namentlich in der älteren Literatur gerne dargestellt ist. Die häufigen Totwässer sind, wie schliesslich jede biologische Untersuchung eines Fliessgewässers zeigt, auch für Tiere bewohnbar, welche ausser ihrem rheophilen Verhalten keine weiteren Merkmale einer Anpassung an das fliessende Wasser besitzen (am schönsten demonstriert durch Gammarus), und selbst die exponiertesten Stellen, wo die Strömung ungehindert angreifen kann, sind dank der Ausbildung der Grenzschichten alles andere als unbewohnbar; es erübrigt sich, dafür nochmals Beispiele anzuführen.

\section{Chemische Besonderheiten}

Der Titel dieses Referates sieht vor, dass wir uns auch mit der Wirkung der Strömung auf die chemischen Verhältnisse im fliessenden Wasser befassen. Während Strömung und Chemie der Gewässer wenig gemeinsam haben und einander nur in Spezialfällen, deren Aufzählung uns kaum etwas nützen würde, beeinflussen, so sind es aber wiederum die Vorgänge im Kleinen, im Totwasser, in der Grenzschicht, welche einige Ưberlegungen rechtfertigen. Allerdings können wir uns hier nicht auf experimentelle Arbeiten stützen; die folgenden Gedanken sind einstweilen Theorie.

Mit einer Reihe von Versuchen an Insektenlarven haben wir seinerzeit festgestellt, dass die Intensität ihres Stoffwechsels vom Sauerstoffgehalt des Wassers abhängt (bei konstanter Temperatur) und auch von der Gescbwindigkeit, mit der es am Körper des Tieres vorbeifliesst [I], und zwar trifft das zur. Hauptsache für jene Formen zu, welche keine eigenen Ventilationsorgane besitzen. Ähnliche Ergebnisse wurden von anderen Autoren schon früher erhalten, und das gleiche gilt grundsätzlich auch für den Stoffwechsel der Wasserpflanzen, wie GESSNER [3, Seite $20 \mathrm{rff}]$ ausführlich dargestellt hat. Um diese Erscheinung zu verstehen, müssen wir uns erneut vergegenwärtigen, dass sich um jeden 
Körper herum, also auch um Tiere und Pflanzen, eine Grenzschicht ausbildet, nicht nur im freien Wasser, sondern natürlich auch in Totwasserzonen. Jedes Lebewesen ist eingehüllt von seiner eigenen, mehr oder weniger dicken Grenzschicht, von der es wie von einem dünnen, anhaftenden Hof umgeben ist.

Der Stoffaustausch nun, welcher bewirkt, dass die Stoffwechselprodukte der Organismen weggeführt werden und das verbrauchte Wasser dauernd erneuern, ist ein rein mechanischer Vorgang, bewirkt durch die Turbulenz. Er greift aber nicht bis zur Körperoberfläche des betreffenden Organismus, sondern nur bis zu seiner Grenzschicht; diese wird von der Wasserströmung ja viel langsamer erfasst. Die Grenzschicht, in welcher normalerweise laminare, stark verlangsamte Strömung herrscht, ist somit ein wesentliches Hindernis für den Austausch. In seiner letzten Phase kann er deshalb nur noch durch Diffusion erfolgen. Je rascher nun das Wasser fliesst, um so dünner wird auch die Grenzschicht, und umso leichter werden sich der Gas- und Stoffaustausch und damit auch die Atmung vollziehen können. Ein Tier zum Beispiel kommt somit mit dem fliessenden Wasser nicht in unmittelbare, direkte Berührung, sondern nur mit dem Wasser seiner eigenen Körpergrenzschicht. Es untersteht deshalb nicht dem Sauerstoffgehalt seiner Umgebung, sondern dem jeweiligen Gehalt seines von der Grenzschicht festgehaltenen Diffusionshofes. Dieser Sauerstoffgehalt ist aber das momentane Ergebnis des Zusammenspiels mehrerer Komponenten, unter denen die Strömungsgeschwindigkeit eine massgebliche Rolle spielt. Je dünner die Grenzschicht ist, um so rascher wird sich der verbrauchte Sauerstoff erneuern, das heisst, um so höher ist der Sauerstoffgehalt, mit dem das Tier in Berührung kommen kann, und um so intensiver ist auch der Stoffwechsel, wenigstens bei Tieren, die mit Tracheenkiemen atmen.

Mit diesen spekulativen Úberlegungen, welche sich jedoch von den Erfahrungen zahlreicher Atmungsmessungen herleiten, ist noch nicht viel bewiesen; ein weites Arbeitsfeld liegt hier noch vor uns.

Zum Schluss sei ein Punkt noch besonders herausgestellt: In den vorangegangenen Seiten dieses Referates haben wir uns mit den wichtigsten Erscheinungen des fliessenden Wassers auseinandergesetzt. Es handelt sich hier um Tatsachen, die indessen wichtig und bedeutsam sind, um triviale Naturerscheinungen zwar, die jedoch das Leben im Fliesswasser 
in einem dermassen weiten Bereich beeinflussen und bestimmen, dass man sich bemühen sollte, sie nicht nur im jetzigen Augenblick zu verstehen, sondern dass man sich von diesen ja unsichtbaren Vorgängen eine konkrete Vorstellung macht und sich diese Vorstellung tiefer eingehen lässt, sie sich zu eigen macht im Empfinden und Gefuihl, um damit eine der Grundlagen für das Verständnis der Bewegungsvorgänge im natürlichen Gewässer zu erhalten.

\section{LITERATURVERZEICHNIS}

[I] АмвӥнL, H., Die Bedeutung der Strömung als ökologischer Faktor. Schweiz. Z. Hydrol. 21, 133 (I959).

[2] Edington, J. M., und Molyneux, L., Portable mater velocity meter. J. Scientific Instruments 37,455 (1960).

[3] Gessner, F., Hydrobotanik, I. Energiebausbalt (Berlin 1955).

[4] JAAG, O., Die Kryptogamenflora des Rbeinfalls und des Hocbrbeins von Stein bis Eglisau. Mitt. Natf. Ges. Schaffhausen 14, I (1938).

[5] Macan, T. T., Factors that limit the range of fresbwater animals. Biological Reviews 36, I5 1 , (I96I).

[6] Pleskot, G., Der Stand der biologiscben Fliesswasserforscbung. Verh. dtsch. Zoologen Mainz 1949, p. 278.

[7] PRANDTL, I., Fübrer durcb die Strömungslebre (Braunschweig I949).

[8] RutTNer, F., Bemerkungen über den Sauerstoffgehalt der Gewässer und dessen respiratorischen Wert. Naturwissenschaften 14, I237 (I926).

[9] RutTner, F., Grundriss der Limnologie (Berlin I962).

\section{RÉSUMÉ}

\section{Particularités des courants des points de vue physico-chimiques et biologiques}

\section{Courants laminaires et turbulents}

L.e courant laminaire n'existe pratiquement pas dans la nature, du moins pas dans les eaux de surface; c'est seulement dans les eaux souterraines (et donc aussi dans le psammon) que le courant laminaire a une grande importance. Les conditions qui permettent la laminarité se rencontrent seulement dans les eaux souterraines, tandis qu'elles sont matériellement impossibles dans les eaux superficielles. Au delà d'une certaine vitesse qui dépend des dimensions et de la forme du canal et qui, en tout cas, est très basse, le courant laminaire devient instable; la masse d'eau se désagrège en une infinité de petits paquets d'eau qui se meuvent en tous sens. 
Les inégalités du radier et des berges font naître des tourbillons - le courant est devenu turbulent. Il est caractérisé par le mélange intensif de toute la masse d'eau. Quoiqu'il puisse y avoir des tourbillons, la turbulence d'un courant ne dépend en principe pas des tourbillons, contrairement à l'opinion courante.

La turbulence est la base mécanjque pour tout échange de substances dans l'eau. Sans la turbulence, l'échange ou la compensation entre les différentes concentrations de substances dissoutes ne pourraient se faire que par diffusion. Toutefois, sous de telles conditions, il n'y aurait sans doute pas de vie dans l'eau. La turbulence, resp. la compensation qui est rendue possible grâce à la turbulence, est par conséquent une des bases physiques de la vie aquatique, non seulement dans l'eau courante, mais aussi dans les lacs. A vrai dire, cet échange est limité dans une certaine mesure par un autre phénomène physique, les couches limite.

\section{Couches limite}

La couche limite est la couche d'eau ralentie qui se forme à proximité immédiate de tout corps solide stationnaire. Elle n'est ni fixe, ni immobile, mais forme la zone de transition entre le courant d'eau libre et le substratum. Les conditions qui règnent ici sont principalement laminaires. Si la limnologie s'est, jusqu'ici, peu occupée de ces couches limite, c'est sûrement parce qu'on de disposait pas de procédés de mesure appropriés et suffisamment simples. Pour nos propres recherches, nous avons rendu le courant visible en petits canaux, au moyen d'une méthode optique et pu ainsi mesurer également des couches limite. Nous avons ainsi appris que l'épaisseur des couches limite, même sous des conditions extrêmes, est de l'ordre de grandeur de I à plusieurs millimètres. C'est justement la sphère où se déroule la vie dans l'eau courante. La couche limite a donc une très grande importance biologique. Les petits organismes qui vivent dans les ruisseaux en profitent, ainsi que les formes plates lithophiles et surtout aussi minuscules larves des éphéméroptères. De plus, cette couche a de l'importance pour la formation de colonies d'algues dans le substratum nu.

\section{Eau morte}

On peut également citer l'eau morte parmi les phénomènes propres aux eaux courantes. On appelle ainsi les zones fortement ralenties et séparées du courant normal qui se trouvent derrière les pierres, derrière chaque élévation dans l'eau, après tout resserrement du profil d'écoulement, etc. Ces zones d'eau morte recouvrent une large partie du radier. C'est pourquoi une partie également grande de la vie rhéophile se trouve, non dans le courant lui-meme, mais dans l'eau morte, c. à. d. à l'abri du courant.

\section{Conditions cbimiques}

Le courant turbulent agit en tout cas favorablement sur le chimisme, en particulier sur l'échange de gaz et de substances des organismes aquatiques; ceci explique aussi «l'abondance physiologique en nourriture et en oxygène» de l'eau courante (RutTNer). Ces échanges ne se font cependant pas tout à fait librement, car ils sont partiellement entravés par la couche limite. Des conditions laminaires règnent dans cette couche limite qui se forme naturellement aussi autour de tous les orga- 
nismes; un échange n'est donc possible ici que par le moyen de la diffusion. L'épaisseur de la couche limite dépend essentiellement de la vitesse du courant qui la dépasse. Par conséquent, la vitesse de l'échange, resp. de renouvellement d'eau de la couche limite d'un animal ou d'une plante, dépend de la vitesse à laquelle l'eau coule. I es expériences faites sur des larves d'insectes ont en tout cas montré que la respiration diminue lorsque le courant devient moins rapide.

Tous ces différents aspects de l'eau courante - la turbulence en général avec les échanges comme conséquence principale, l'eau morte et les couches limite - influencent toute la vie aquatique dans l'eau courante. Cependant, étant donné la difficulté de voir ces phénomènes, il est également difficile de bien les saisir et seule une repréaentation claire, concrète et aussi approfondie que possible pourra nous aider à sssigner au courant la place qui lui revient justement parmi les facteurs écologiques.

Separatdruck aus der Schweiz. Z. Hydrologie 24, Fasc. 2, 1962. Herausgegeben mit Unterstützung der Stiftung der Wirtschaft zur Förderung des Gewässerschutzes in der Schweiz. 